\title{
Lidil
}

Revue de linguistique et de didactique des langues

\section{Langue et études de la langue, Approches linguistiques et didactiques}

2004, Claude vargas (dir.), Aix-en-Provence, Publications de l'université de Provence.

Francis Grossmann

\section{OpenEdition}

\section{Journals}

Édition électronique

URL : http://journals.openedition.org/lidil/160

DOI : $10.4000 /$ lidil. 160

ISSN : 1960-6052

Éditeur

UGA Éditions/Université Grenoble Alpes

\section{Édition imprimée}

Date de publication : 1 juin 2005

Pagination : 205-210

ISBN : 2-914176-12-0

ISSN : $1146-6480$

Référence électronique

Francis Grossmann, « Langue et études de la langue, Approches linguistiques et didactiques », Lidil [En ligne], 31 | 2005, mis en ligne le 03 octobre 2007, consulté le 21 septembre 2020. URL : http:// journals.openedition.org/lidil/160 ; DOI : https://doi.org/10.4000/lidil.160

Ce document a été généré automatiquement le 21 septembre 2020.

(C) Lidil 


\title{
Langue et études de la langue, Approches linguistiques et didactiques
}

\author{
2004, Claude vargas (dir.), Aix-en-Provence, Publications de l'université \\ de Provence.
}

Francis Grossmann

1 Il n'est jamais commode de rendre compte d'un ouvrage collectif, surtout lorsqu'il aborde, comme c'est le cas ici, un ensemble très vaste de questions liées à ce que l'on nommait autrefois, sans trop d'état d'âme, la grammaire, et dont la dénomination même, comme l'objet, semblent devenir aujourd'hui problématiques. C'est sans doute le premier mérite de l'ouvrage, issu d'un colloque qui s'est tenu à Marseille en juin 2003, de faire écho aux interrogations et aux hésitations qui traversent le champ de la didactique $d u$ français, langue première (et pour certaines contributions, langue seconde ou étrangère) au sujet de cet objet d'enseignement devenu incertain. Je me limiterai donc à présenter - trop rapidement - quelques unes des contributions, en assumant le caractère subjectif de mon choix, et en m'excusant auprès des autres auteurs de ne pouvoir faire ici, faute de place, un compte-rendu exhaustif. Un premier ensemble de contributions s'efforce précisément de réfléchir, au statut disciplinaire des objets d'enseignement (qu'ils soient coiffés de l'étiquette de "grammaire » ou de "syntaxe ») et au nouveau positionnement qu'ils occupent dans l'enseignement du français. Ainsi, Jean-François Halté, après avoir rappelé les difficultés d'une articulation cohérente des niveaux phrastiques, énonciatifs et discursifs défend-il l'idée que le salut - en l'occurrence la reconquête d'une identité didactique lisible - passe, pour la grammaire, par un liaison plus claire aux activités de production et de réception. Il s'agirait alors d'orienter les activités grammaticales vers la «construction des savoir faire langagiers ", en évitant la tentation, qu'il juge prématurée, de recourir à une théorie totalisante. Claude Vargas, insiste plutôt, à l'inverse, sur la nécessaire cohérence de l'ensemble, en recourant à la notion de recomposition didactique, qu'il préfère à celle de transposition. Il propose un programme volontariste et peut-être un 
brin optimiste : aucune définition, liée à un certain niveau ou à un point de vue sur la langue, ne devrait entrer en contradiction avec ce qui est proposé par ailleurs à un autre niveau d'analyse. Bernard Combettes propose quant à lui une mise en perspective salutaire, et des directions pour l'avenir: il convient, selon lui, de relativiser aujourd'hui des oppositions comme celles de grammaire de phrase et de grammaire de texte, pour s'intéresser davantage aux opérations elles-mêmes. Il est temps, nous dit-il, d'étudier pour elles-mêmes des notions telles que celles de prédication, de détermination (opposée à caractérisation), tandis qu'elles n'apparaissent aujourd'hui qu'incidemment dans les grammaires scolaires. Il s'agirait alors de subordonner l'étude traditionnelle des parties du discours à cet objet principal. De même, le rejet, justifié historiquement des confusions qu'entretenait la grammaire traditionnelle entre les niveaux syntaxiques et sémantiques doit aujourd'hui céder la place à une approche raisonnée des relations entre sémantique et syntaxe, repérables notamment aux niveaux des relations actancielles et de la détermination nominale. On ne peut que souscrire à un tel programme, même si sa faisabilité reste conditionnée par des propositions didactiques plus précises, comme le reconnaît l'auteur. Les autres contributions de cette première section, consacrée à l'étude de la langue, abordent un grand nombre de questions, et selon des angles divers. Mireille Bilger et Paul Cappeau, à partir de plusieurs exemples, montrent tout l'intérêt qu'il y a, pour l'étude de la langue, à se fonder sur des corpus, et en particulier des corpus véritablement représentatifs de l'ensemble de la langue. Cet appui permet en particulier de fournir des indications sur la productivité des phénomènes étudiés, ce que ne permettent généralement pas les grammaires. Belinda Lavieu, reprenant l'opposition classique entre « compléments de verbe » et " compléments de phrase » exprime la nécessité d'une approche plus précise, et reprend à son compte la typologie de Delaveau (2001) qui oppose d'abord compléments sous-catégorisés (argumentaux) et circonstanciels (ou modifieurs), puis distingue, dans cette deuxième classe, entre compléments de verbes (il a annoncé sa démission à la radio) et compléments de phrase (il a annoncé sa démission à l'aube). Elle présente ensuite les tests qui permettent de distinguer entre les différents compléments. Ce type d'étude est très utile : il permet d'éviter le découragement qui envahit parfois les enseignants : oui, il est possible en faisant avancer la réflexion théorique, d'échapper au malaise lié à l'imprécision des catégories, et aux frontières mal tracées. Jean-François de Pietro poursuit son combat salutaire, visant à ouvrir la réflexion grammaticale à d'autres langues, ainsi qu'aux dialectes que les élèves apportent avec eux. Il souligne l'intérêt que peut présenter ce « détour par d'autres langues " pour modifier la posture des élèves face aux langues et au langage. JeanClaude Chabanne, de son côté, réagissant aux orientations des dernières Instructions officielles, se demande si « la grammaire est entièrement soluble dans les pratiques d'écriture ». S'il semble incliner vers l'idée que certaines tâches d'écriture peuvent être analysées comme des situations d'apprentissage de la langue, il montre aussi le prix à payer, en terme de formation d'enseignants, puisque de telles pratiques sont associées à des "gestes professionnels complexes". Plusieurs études montrent l'intérêt de prendre un peu de recul - historique en particulier devant les "nouveautés» introduites dans l'enseignement de la langue : c'est le cas de la contribution de Guy Legrand, qui fait une intéressante synthèse des contenus de l'enseignement grammatical selon les textes officiels, depuis 1871 ; c'est aussi le propos de Monique Lebrun et de Priscilla Boyer, qui analysent les programmes français et québécois de ces 
quarante dernières années. Anita Tcherkeslian-Carlotti propose une réflexion sur la notion très polysémique de « discours » et son utilisation dans les manuels scolaires.

2 La seconde section de l'ouvrage s'intéresse plus directement à la question des apprentissages, et déborde largement le territoire traditionnel de la grammaire. Il s'agit par exemple d'examiner la manière dont les élèves peuvent entrer dans une posture métalinguistique (Fabienne Calame-Gippet) ou bien de mieux prendre en compte les langues enseignées pour développer les compétences métalinguistiques des élèves (Marielle Rispail) : cette deuxième étude fait ainsi écho aux propositions de de Pietro en montrant l'intérêt que peut présenter l'étude d'une langue minoritaire (en l'occurrence le provençal) pour permettre d'accéder aux concepts linguistiques. Deux contributions abordent frontalement la question des relations entre langue et orthographe. La contribution de Véronique Rey et de Carine Sabater part du postulat que «l'écriture représente l'aboutissement de l'apprentissage de l'écriture, et non le processus développemental de réflexion sur le développement de la langue, à ses différents niveaux ». Dans le cadre de ce modèle, les correspondances phonèmesgraphèmes ne suffisent pas dans l'apprentissage de l'écrit, il y a nécessité aussi d'apprendre à manier les correspondances morphèmes-mots. Catherine Brissaud et Danièle Cogis, de leur côté, s'intéressent à un point particulier de l'apprentissage orthographique: la reconnaissance du sujet grammatical. Appuyé sur des données précises, leur article débouche sur une conclusion didactique qui inverse, de manière heureuse, la thèse de Chervel : la grammaire scolaire n'est plus vue exclusivement au service de l'orthographe ; c'est l'orthographe qui est au service de la grammaire. MarieNoëlle Roubaud et Yvonne Touchard montrent quant à elles le long chemin que doit parcourir l'enfant, pour aboutir, vers sept ans, à construire la notion de verbe, dans toutes ses dimensions.

3 Cette question du verbe, et de son repérage, est reprise, dans la troisième section de l'ouvrage (consacrée aux «pratiques»), par François Quet et Dominique Dourojeanni. Fruit d'un travail de terrain, leur contribution montre la difficulté qu'ont les élèves à entrer dans une activité réflexive sur la langue et fournit des pistes concrètes pour les aider à y parvenir. Micheline Cellier présente quant à elle quelques dispositifs permettant de "dire l'orthographe». Elle expose le travail d'une équipe INRP s'intéressant au rôle que peuvent jouer les interactions orales pour la construction de savoirs linguistiques, en particulier orthographiques. Dans une perspective voisine, Josette Isidore-Prigent revient sur les "ateliers de négociation graphique" expérimentés depuis de nombreuses années par le Groupe de Recherche en Didactique de l'Orthographe, à l'Université de Bourgogne. G. Teste suggère quelques pistes permettant aux élèves démobiliser leurs connaissances orthographiques en situation de production écrite. Corinne Gomila s'intéresse, de son côté, à la place des savoirs grammaticaux dans l'enseignement de la lecture au CP. A contre-courant de certaines tendances actuelles, elle insiste sur la nécessité d'une explicitation des concepts grammaticaux nécessaires à l'apprentissage, l'évitement du métalangage risquant de poser problème, principalement aux élèves en difficulté. En contrepoint de ces études portant sur des faits de langue très spécifiques, Daniel Bessonnat et Danielle Coltier rendent compte, non sans humour, d'une année de travail, en classe de 3e, autour de la notion d'ironie.

4 Pour finir, une dernière section est consacrée aux représentations : Monique Lebrun et Colette Baribeau insistent sur le rôle des représentations de la langue chez les futurs 
maîtres, et confirme ce que d'autres études nous ont déjà montré, à savoir l'importance que revêt chez eux la vision normative, avec une tension entre l'oral (peu normé) et l'écrit (surnormé). C'est cette même question des modèles intériorisés que traite AnneCatherine Oudart, mais en l'orientant cette fois vers des adultes en formation, dans le cadre de l'apprentissage d'écrits professionnels. Carole Fisher tente de préciser la place des représentations des apprenants en didactique de la grammaire. De manière plus générale, elle propose de "lier davantage l'étude des représentations à la mise à jour des obstacles à l'apprentissage, en dépassant une perspective trop proprement descriptive ». Etant donné l'usage proliférant de cette notion de représentation, il serait utile d'aboutir, nous dit-elle, à une définition plus précise du concept dans le champ didactique. Martine Fialip-Baratte, s'appuyant sur le concept de « rapport à l'écriture ", théorisé par Christine Barré-De Miniac, présente les résultats de son travail de thèse, en montrant les représentations que se font des enfants de maternelle du lireécrire en montrant que certaines d'entre elles peuvent se révéler des obstacles.

5 Au total, l'ouvrage a les qualités et les défauts du genre "Actes de colloque »: on y trouve à boire et à manger, et le partage en sections reste en définitive assez artificiel. L'« étude de la langue » y est vue comme s'identifiant à l'enseignement grammatical, au sens classique du terme, mais l'ouverture du côté de l'orthographe, de l'écriture et même de l'oral y est sensible. Du coup, les problématiques abordées sont tellement diverses qu'il est parfois difficile de trouver les fils qui peuvent conduire de l'une à l'autre. Cependant, étant donné la grande qualité de certaines des contributions proposées, ce livre mérite en tout état de cause de faire partie de la bibliothèque, sinon de l'honnête homme, du moins du didacticien-linguiste.

\section{AUTEUR}

\section{FRANCIS GROSSMANN}

Université Stendhal-Grenoble 3, Laboratoire Lidilem. 